\title{
A LAGARTA DE CERCONOTA ACHATINA (ZELLER) (LEPIDOPTERA, OECOPHORIDAE, STENOMATINAE): BIOLOGIA E OCORRÊNCIA EM PLANTAS HOSPEDEIRAS DO GÊNERO BYRSONIMA RICH (MALPIGHIACEAE)
}

\author{
I. Andrade 1,3 \\ Ivone R. Diniz ${ }^{1}$ \\ H.C. Morais ${ }^{2}$
}

\begin{abstract}
THE LARVA OF CIRCONOTA ACHATINA (ZFILIIR) (LIPIDOPTIRA. STINOMATINAE, OLEOPHORIDAL:): BIOLOGY AND OCCURRINCE ON FOOD PIANTS OF THI GENIS BYRSONMA RICH (MALPIGIIACTAR:). Caterpillars of Cerconota achatina (Zeller, 1855) feed on the leaves of Byrsonima Rich in cerrado (savanna) of Brasilia (Distrito Federal). They build shelters by tying leaves together with silk and frass. Within the leaf shelters the caterpillars form a tube of silk in which they feed. develop and pupate. In the laboratory the pupal stage is 14.4 days $(\mathrm{sd}=8.6: \mathrm{n}=46$ ). It was not possible to determine the age or numbers of instars of caterpillars in the wild. Of 93 caterpillars collected. $6(0) \%$ were feeding on B. coccolobifolia (Spr.) Kunth. $25 \%$ on $B$. crassa Nied. and $15 \%$ on B. verbascifolia [Rich. ex| Adr.\& Juss. These numbers suggest a preference for $B$. coccolobifolia. Caterpillars of all size were found in every month of the year which suggest ovelapping generations and multivoltinism. They did not show a preference for new foliage and $75 \%$ of them were found on host-plants on wich less than $25 \%$ of leaves were young.

KEY WORDS. Lepidoptera. Oecophoridae. Cerconota achatina. Malpighiaceae. Byrsonima
\end{abstract}

A fauna de Lepidoptera do cerrado é muito rica havendo estimativas de mais de 900 espécies de borboletas para o planalto central (BROWn \& MiELKE 1967) e de 5000 a 8000 espécies de mariposas para a região de cerrado (BECKER 1991). No entanto, muito pouco se conhece sobre a biologia desses organismos.

Este trabalho apresenta dados sobre a biologia de Cerconota achatina (Zeller, 1855), uma mariposa da América do Sul, comum na região de cerrado, que se alimenta de folhas de murici (Bvrsonima sp.).

A subfamília Stenomatinae é um grupo de microlepidópteros rico em espécies nas Américas, especialmente na região tropical, ocorrendo também em Madagascar, sudeste asiático e região Australiana (DUCKWORTH 1973). Nas Américas é representada por mais de 1200 espécies, distribuídas desde os Estados Unidos até a Argentina (AIELlo 1981). O gênero Cerconota Meyrick, 1915 é

1) Departamento de Zoologia. Universidade de Brasilia, 7(091()-90(0) Brasília. Distrito Federal, Brasil

2) Departamento de Ecologia, Universidade de Brasília, 70910-90() Brasília, Distrito Federal, Brasil.

3) Curso de Pós-Graduação en Ecologia. Universidade Estadual de Campinas. Caixa Postal 6109. 13081-97() Campinas, Sào Paulo, Brasil.

Revta bras. Zool. 12 (4): $735-741,1995$ 
citado para o México, Panamá, Costa Rica, Colombia, Venezuela, as Guianas, Bolívia e Brasil (Amazonas, Pará e Santa Catarina) por BECKer (1984) que apresenta a sinonimia e a combinação atual para o binômio Cerconota achatina.

Em um levantamento qualitativo de lagartas em plantas de um cerrado de Brasília, realizado entre 1991 e 1993, foi encontrado Cerconota sciaphilina (Zeller, 1855) em Roupala montana Aubl. (Proteaceae) e Eryotheca pubescens (Mart.\& Zucc.) (Bombacaceae) e Cerconota achatina em Byrsonima coccolobifolia (Spr.) Kunth e B. crassa Nied. (Malpighiaceae) (DinIZ \& MoraIS no prelo).

\section{MATERIAL E MÉTODOS}

O levantamento de larvas em Byrsonima coccolobifolia (Spr.) Kunth, $B$. crassa Nied. e B. verbascifolia (Rich.) Adr. \& Juss. foi realizado na Fazenda Água

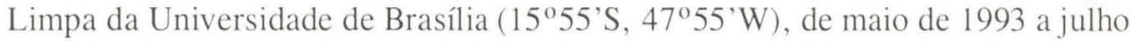
de 1994. A vegetação da Fazenda é descrita por RATTER (1991) e a área de trabalho é a mesma utilizada em Diniz \& MORAIS (no prelo).

Uma parcela de aproximadamente 1 ha de cerrado sensu stricto foi dividida em quadrantes, vistoriados na forma de rodízio (um quadrante a cada semana). Geralmente foram examinados 15 indivíduos de cada espécie de planta por semana, à procura de lagartas. Cada planta foi cuidadosamente examinada e as lagartas encontradas foram coletadas e levadas ao laboratório em sacos plásticos individuais com o nome da planta hospedeira. Para cada planta examinada foi estimada a porcentagem de folhas novas presentes.

No laboratório, as lagartas foram criadas em potes forrados com papel toalha e fechados com filó, recebendo como alimento folhas da espécie hospedeira. As folhas para alimentação eram mantidas em sacos plásticos na geladeira e eram renovadas nos potes de criação 3 vezes por semana, onde eram mantidas com o pecíolo envolto em algodão úmido. As datas nas quais as lagartas empuparam e tornaram-se adultos foram anotadas individualmente. A identificação da espécie foi feita pelo Dr. Vitor O. Becker (Empresa Brasileira de Pesquisa Agropecuária).

\section{RESULTADOS E DISCUSSÃO}

\section{Biologia de CERconota achatina}

$\mathrm{O}$ adulto de Cerconota achatina é uma pequena mariposa de coloração escura que possui entre 1,5 e $2 \mathrm{~cm}$ de envergadura das asas (Fig. 1a).

A lagarta é cilíndrica de cor castanho-avermelhada com a cápsula cefálica castanho escuro (Fig. 1b). Em seu último ínstar, atinge cerca de três centímetros de comprimento e então constrói um abrigo mantendo justapostos os limbos de duas folhas, prendendo-os com fios de teia. Dentro deste abrigo tece um fino tubo de teia entre as superfícies das duas folhas. A lagarta movimenta-se para a frente e para trás dentro do tubo, ao lado do qual se acumulam fezes (Fig. 1c).

Não foram encontradas lagartas de $C$. achatina fora de seus abrigos. Se é retirada do abrigo realiza rápidos movimentos de contorção (pulos). Estes movi- 
mentos podem funcionar como defesa contra predação (FREITAS \& OLIVEIRA 1994) e muitas vezes cai no chão após a abertura de um abrigo.
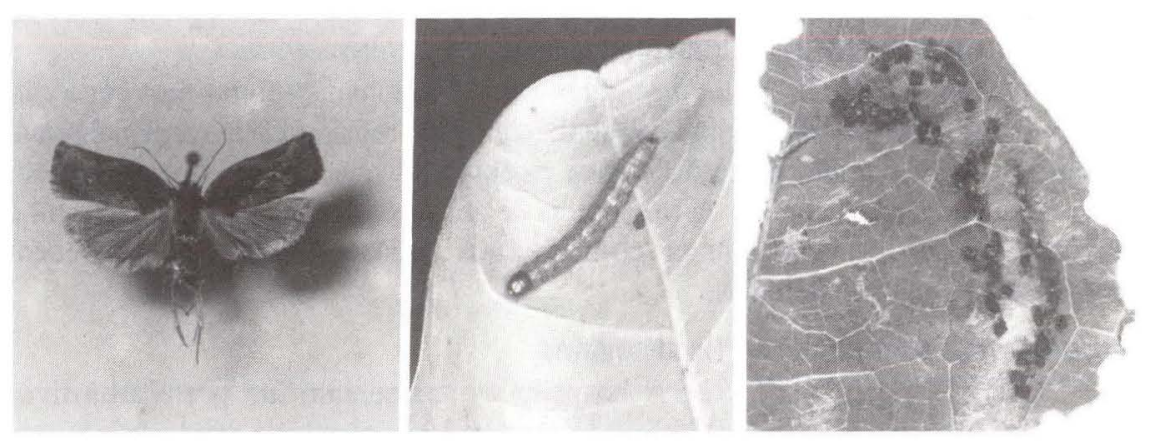

Fig. 1. Cerconota achatina. Da esquerda para a direita: adulto, lagarta e lagarta em seu abrigo, evidenciando o tubo de teia e fezes.

Cerconota achatina alimenta-se da folha na qual constrói o tubo, colocando uma parte do corpo para fora deste e raspando a superfície da folha. Depois que a folha envelhece a lagarta reúne, com teia, outras folhas ao abrigo formando um aglomerado de folhas. No campo, foram encontradas lagartas em folhas bastante velhas e unidas à planta por fios de teia. No laboratório, constroem novos abrigos e tubos sempre que os anteriores são destruídos. Quando apenas uma folha é mantida no pote, as lagartas ou fixam-na à superfície do pote ou utilizam o papel toalha para a construção do abrigo. Em qualquer destes casos elas tecem o tubo de teia dentro do abrigo.

Foram coletados 93 abrigos com lagartas. Em 80 deles havia apenas uma lagarta, em dez havia duas e em três havia quatro lagartas. Nos casos de abrigos com mais de uma lagarta, estas tecem tubos individuais apesar de utilizarem as mesmas folhas como substrato e como alimento.

A lagarta de $C$. achatina empupa dentro do tubo de seda permanecendo, portanto, entre as folhas do abrigo. No laboratório, a duração do estágio de pupa foi de 14,4 dias $(s=8,6 ; n=46)$. Não há informações sobre a idade da lagarta quando coletada e sobre o número e duração de cada ínstar, mas o tempo de permanência como lagarta no laboratório tende a ser longo. Considerando o período entre a data de coleta e a data de pupa elas permaneceram em média 53,2 dias no laboratório $(\mathrm{s}=21,2 ; \min .=7$ e $\max .=95 ; \mathrm{n}=47)$.

Três lagartas $(n=112)$ morreram em decorrência de parasitismo. Os parasitóides (Hymenoptera) emergiram da pupa. Exemplares dos himenópteros foram enviados a especialistas para identificação.

A construção de abrigos é um fenômeno comum em microlepidópteros, especialmente entre as famílias Oecophoridae, Tortricidae e Gelechiidae (CAPPUCCINO 1993). A literatura mostra que abrigos proporcionam microhabitat 
favorável (HUNTER \& EISNER 1989), um aumento na qualidade da folha de alimentação (SAGER 1992) e proteção contra predadores e parasitóides (DAMMAN 1987; CAPPuCCino 1993). A utilização de um mesmo abrigo por mais de uma lagarta (co-habitação), por outro lado, traz desvantagens como a redução da qualidade alimentar da folha pelo aumento da herbivoria e o acúmulo de fezes, o que pode influir no desenvolvimento larval (CAPPUCCINO 1993).

Os abrigos de $C$. achatina podem estar funcionando como uma proteção contra dissecação em uma região com baixa umidade relativa do ar, especialmente durante a seca, e como proteção contra parasitóides que foram encontrados em uma freqüência muito baixa. A freqüência de abrigos com mais de uma lagarta é baixa sugerindo desvantagem na co-habitação. Todos estes aspectos merecem investigações mais detalhadas.

\section{OCORRÊNCIA NAS PLANTAS HOSPEDEIRAS}

As três espécies de plantas hospedeiras apresentam um porte arbustivoarbóreo, sendo que $B$. coccolobifolia é comumente encontrada na forma de arbusto. Byrsonima crassa possui folhas de tamanho médio com pelos na face inferior quando madura e $B$. verbascifolia apresenta folhas grandes e rígidas com muitos pelos em ambas as faces. Byrsonima coccolobifolia apresenta folhas menores, mais macias e com baixa densidade de pelos. As plantas são caducifolias, trocando suas folhas durante a seca de forma assincrônica entre os indivíduos. Informações sobre a fenologia dessas espécies são fornecidas em BARROS (1992) e MORAIS et. al. (1995).

Foram vistoriadas 1608 plantas e em 321 (20\%) delas foram encontradas lagartas de diferentes espécies. Cerconota achatina apresentou o maior número de ocorrências tendo sido coletada em $93(6 \%)$ das plantas vistoriadas (Tab. I). A freqüência de ocorrência de $C$. achatina foi maior em $B$. coccolobifolia $(60 \%$ das ocorrências) do que em $B$. crassa ( $24 \%$ ) e em $B$. verbascifolia (15\%), indicando uma preferência entre as plantas hospedeiras deste gênero na área de estudo. Este padrão é constante ao longo do ano, sendo sempre maior o número de ocorrências de $C$. achatina em B. coccolobifolia. Essa lagarta representou $47 \%$ da fauna associada a $B$. coccolobifolia. A proporção cai para $23 \% \mathrm{em} B$. crassa e $14 \% \mathrm{em}$ B. verbascifolia.

Tabela I. Número de plantas hospedeiras do gênero Byrsonima vistoriadas e de plantas com lagartas, entre maio/93 e julho/94, em uma área de cerrado em Brasilia (Distrito Federal). (PL) número de plantas com lagartas de qualquer espécie, (PC) número de plantas com lagartas de Cerconota achatina.

\begin{tabular}{lccc}
\hline \multicolumn{1}{c}{ Espécie de planta } & Plantas vistoriadas & $\mathrm{PL}$ & $\mathrm{PC}$ \\
\hline Byrsonima coccolobifolia & 497 & 118 & 55 \\
Byrsonima crassa & 574 & 105 & 24 \\
Byrsonima verbascifolia & 527 & 98 & 14 \\
\hline Total & 1608 & 321 & 93 \\
\hline
\end{tabular}


Cerconota achatina é uma espécie multivoltina, tendo ocorrido em todos os meses do ano (Fig. 2). As lagartas foram mais frequentes na seca (maio a agosto) e no meio da estação chuvosa (janeiro), e foram pouco freqüentes no final da seca e início das chuvas (setembro a dezembro), período que inclui o pico de produção de folhas das três plantas hospedeiras estudadas (Figura 3). Ocorreu uma variação considerável na freqüência de lagartas coletadas nas duas estações secas estudadas (Fig. 3).

\begin{tabular}{|c|c|c|c|c|c|c|c|c|c|c|c|c|c|c|}
\hline B. crassa & $\mathrm{X}$ & $\mathrm{X}$ & $\mathrm{X}$ & & & $\mathrm{X}$ & $\mathrm{X}$ & & $\mathrm{X}$ & & & & $\mathrm{X}$ & $\mathrm{X}$ \\
\hline B. verbascifolia & & $\mathrm{X}$ & & & $\mathrm{X}$ & & & & & & $\mathrm{X}$ & & & $\mathrm{X}$ \\
\hline B. coccolobifolia & $\mathrm{X}$ & $\mathrm{X}$ & $\mathrm{X}$ & $\mathrm{X}$ & $\mathrm{X}$ & & $\mathrm{X}$ & $\mathrm{X}$ & $\mathrm{X}$ & $\mathrm{X}$ & $\mathrm{X}$ & $\mathrm{X}$ & $\mathrm{X}$ & $\mathrm{X}$ \\
\hline & M & $\mathrm{J}$ & $\mathrm{J}$ & A & S & $\mathrm{O}$ & $\mathrm{N}$ & $\mathrm{D}$ & $\mathrm{J}$ & F & M & A & M & J \\
\hline
\end{tabular}

Fig. 2. Ocorrência de lagartas de Cerconota achatina nas três plantas hospedeiras do gênero Byrsonima, em uma área de cerrado durante o período do trabalho.

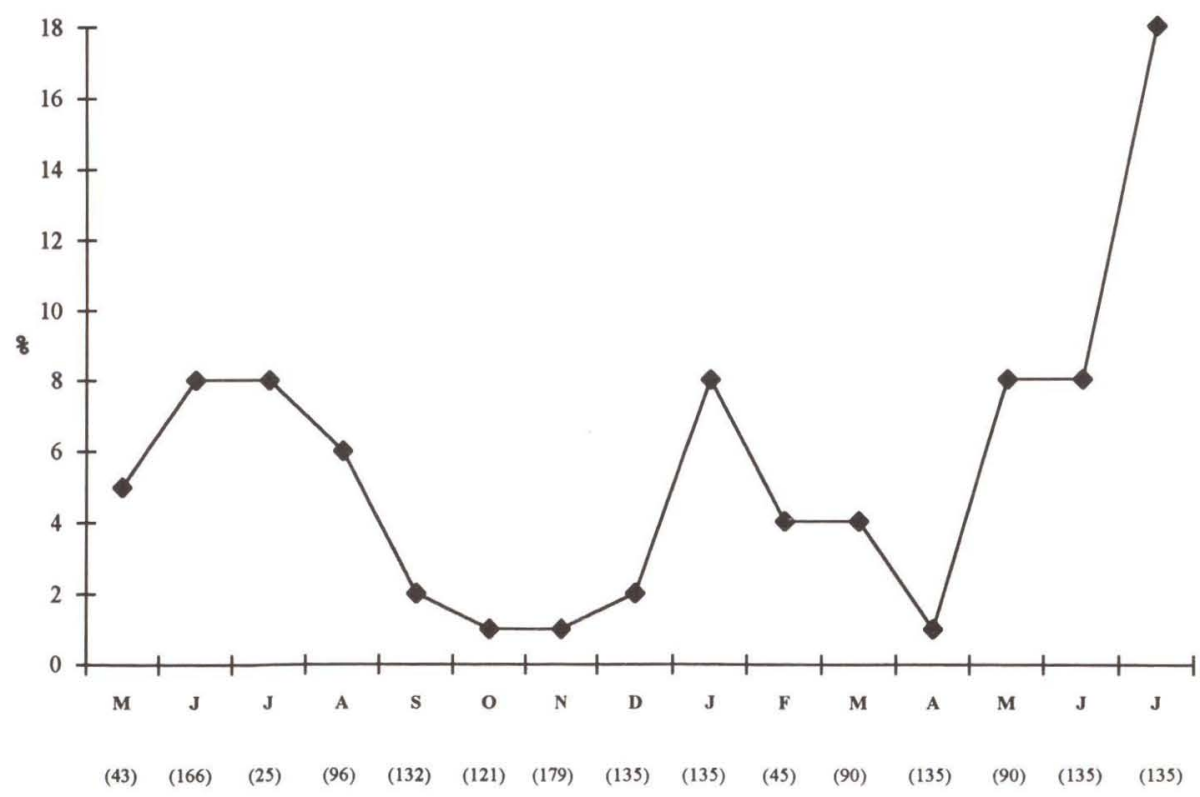

Fig. 3. Freqüência de ocorrência de lagartas de Cerconota achatina em Byrsonima sp. entre maio/93 e julho/94 em um cerrado de Brasília. 
Grande parte das lagartas $(89 \%)$ estava em plantas que apresentavam menos de $25 \%$ de folhas novas. Em $B$. verbascifolia, que apresenta uma densa pilosidade nas folhas novas, todas as lagartas estavam em plantas sem folhas novas, o que, pode estar relacionado com a menor quantidade de pelos nas folhas maduras desta planta.

AGRADECIMENTOS. Agradecemos especialmente ao Dr. Vitor O. Becker pela identificação da espécie, pelas sugestões e correções do texto e por ceder espécimens de sua coleção particular para fotos. A A.P. Dornelles, J. Costa, J.R. Silva, J. Baumgarten, C. Dias e N. Menezes pela colaboração no trabalho de campo e na criação das lagartas; ao Dr. Jader S. Marinho-Filho, F.G Menezes pelas fotos. O trabalho foi parcialmente financiado pelo CNPq (Projeto Herbivoros e herbivoria em cerrado, proc. no. 501025/92-8; bolsa de Iniciação Científica de I. Andrade, proc. no. $800083 / 86-5)$.

\section{REFERÊNCIAS BIBLIOGRÁFICAS}

Aiello, A. 1981. Life history of Antaeotricha sp. (Lepidoptera: Oecophoridae: Stenomatinae) in Panama. Psyche 88 (1-2): 163-168.

BArros, M.A.G. 1992. Fenologia da floração, estratégias reprodutivas e polinização das espécies simpátricas do gênero Byrsonima Rich (Malpighiaceae). Rev. Brasil. Biol. 52 (2): 343-353.

BECKER, V.O. 1984. Taxonomic notes on the Neotropical Microlepidoptera. Revta bras. Ent. 28 (2): 173.

1991. Fauna de lepidópteros dos cerrados: composicão e afinidades com as faunas das regiões vizinhas. I Encontro de Botânicos do Centro Oeste, Brasília, p. 91.

Brown, K.S. \& O.H.H. MielkE. 1967. Lepidoptera of the central Brazil plateau. I. Preliminary list of Rhopalocera (continued): Lycaenidae, Pieridae, Papilionidae, Hesperiidae. J. Lepid. Soc. 21 (3): 145-168.

CAppuccino, N. 1993. Mutual use of leaf-shelters by lepidopteran larvae on paper birch. Ecol. Entom. 18: 287-292.

DAMMAN, H. 1987. Leaf quality and enemy avoidance by the larvae of a pyralid moth. Ecology 68: 88-97.

Diniz, I. R. \& H. C. MORAIS (no prelo). Larvas de Lepidotera e suas plantas hospedeiras em um cerrado de Brasília, DF, Brasil. Revta bras. Ent..

DUCKWORTH, W.D. 1973. The old world Stenomidae: a preliminary survey of the fauna, notes on relationships, and revision of the genus Eriogenes (Lepidoptera: Gelechioidea). Smith. Contr. Zool. 147: 1-21.

FreitAs, A.V.L. \& P.S. OliveirA. 1994. Biology and behavior of the Neotropical butterfly Eunica bechina (Nymphalidae) with special reference to larval defense against ant predation. J. Res. Lep. 31: 1-11.

Hunter, M.D. \& P.G. Willmer. 1989. The potential for inter-specific competition between two abundant defoliators on oak: leaf damage and habitat quality. Ecol. Entom. 14: 267-277. 
Morais, H.C.; I.R. Diniz \& L.C. Baumgarten. 1995. Padrões de produção de folhas e sua utilização por larvas de Lepidoptera em um cerrado de Brasília, DF. Rev. Brasil. Bot. 18: 163-170.

RATTER, J.A. 1991. Notas sobre a vegetação da Fazenda Água Limpa (Brasília, DF). Brasília, Editora Univ. de Brasília, 136p.

SAGERS, C.L. 1992. Manipulation of host plant quality: herbivores keep leaves in the dark. Func. Ecol. 6: 741-743. 